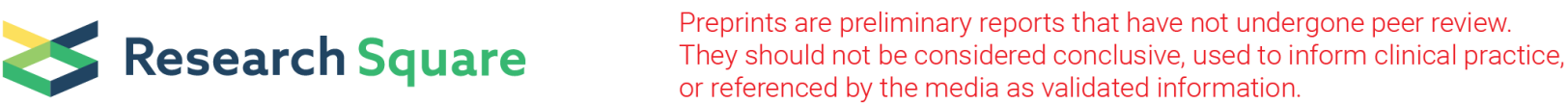

\section{Design Optimization of Nanotube Tunnel Field- Effect Transistor with Bias-Induced Electron-Hole Bilayer}

\section{Xueke Wang}

East China Normal University

Yabin Sun ( $\nabla$ ybsun@ee.ecnu.edu.cn )

East China Normal University https://orcid.org/0000-0002-3783-2885

Ziyu Liu

Fudan University

Yun Liu

East China Normal University

Xiaojin Li

East China Normal University

\section{Yanling Shi}

East China Normal University

\section{Research Article}

Keywords: Band-to-band tunneling, nanotube, electron-hole bilayer, line tunneling, steep switching

Posted Date: September 14th, 2021

DOI: https://doi.org/10.21203/rs.3.rs-869222/v1

License: (1) This work is licensed under a Creative Commons Attribution 4.0 International License. Read Full License 


\section{Abstract}

In this paper, a novel nanotube tunneling field-effect transistor (NT-TFET) with bias-induced electron-hole bilayer (EHBNT-TFET) is proposed for the first time. By the intentional misalignment and an asymmetric bias configuration of the inner-gate and outer-gate, the line tunneling takes place inside the channel, significantly improving the tunneling rate and area. The device principle and performance are investigated by calibrated 3-D TCAD simulations. Compared to the conventional NT-TFET, the proposed EHBNT-TFET exhibits an increased $O N$-state current $\left(I_{O N}\right)$ about 57.2 times and a sub-60 mV/dec subthreshold swing for seven orders of magnitude of drain current. Furthermore, the increased $I_{O N}$ and reduced gate capacitance achieve improved dynamic performance. Compared with conventional NTTFET, the intrinsic delay decreased about 142 times is obtained in EHBNT-TFET.

\section{Introduction}

As feature size scales down constantly, conventional MOSFETs have reached physical limitation. Power dissipation rises considerably due to the high-leakage current and unscalable supply voltage $\left(V_{D D}\right)$ [1]. With the rising demand for mobile phones, lower power is becoming an important requirement and some novel devices have been proposed, such as negative capacitance field-effect transistor, impact ionization metal-oxide-semiconductor transistor and tunnel field-effect transistor [2-7]. The quantum tunneling in tunnel field-effect transistor (TFET) achieves an extremely-low OFF-state current (I IFF) and a steep subthreshold slope (SS) below $60 \mathrm{mV} / \mathrm{dec}$ at room temperature [7], so that $V_{D D}$ could be further scaled down.

However, two major challenges restrict TFET in practical applications. The first one is the difficulty of achieving the highly doped source/channel junction with ultra-steep profile experimentally [8]. Though dopingless TFET provides a solution to avoid the high-temperature doping/annealing processes [9], the $O N$-state current $\left(I_{O N}\right)$ degrades due to the existence of large barrier width between source and gate electrodes [10]. The second one is low $I_{O N}$, caused by poor band-to-band tunneling rate and the point tunneling mechanism. To improve $I_{O N}$ of TFET, several approaches have been proposed, such as low bandgap material engineering [11-12], high-k gate dielectric engineering [13], source pocket engineering [14-15] and line tunneling engineering [16-20]. Bias-induced electron-hole bilayer (EHB) concept was applied to double-gate TFET to induce line tunneling in the channel [17-19]. However, 2-D planar structure is difficult to achieve large $I_{O N}$ with small chip-area [20]. Besides, nanowire TFET (NW-TFET) also presents a great potential for performance enhancement because of the excellent electrostatic control. However, dynamic performance and power dissipation of NW-TFET are bound to be affected, when multiple nanowires are vertically stacked to increase the tunneling area. Compared to NW-TFET, nanotube TFET (NT-TFET) achieves similar electrostatic control by the inner/outer-gate, and offers larger tunneling area and current by hollowed cylindrical shaped nanotube structure [21-24]. However, NT-TFET still suffers from the two challenges mentioned above. 
In this paper, a novel NT-TFET with a misaligned inner/outer-gate, named EHBNT-TFET, is proposed for the first time. With appropriate gate work-function and bias configuration, electron-hole bilayer is induced separately, forming an abrupt $\mathrm{p}^{+}-\mathrm{n}^{+}$junction in the intrinsic channel. As a result, the highly doped source/channel junction with abrupt profile is not required in EHBNT-TFET. Using 3-D device simulation, the advantage of EHBNT-TFET is explored and demonstrated in detail. The line tunneling inside the channel dominants the current, instead of the point tunneling between source and channel. Compared to the conventional NT-TFET, $I_{O N}$ of EHBNT-TFET is found to increase about 57.2 times and keep a high $I_{O N} / I_{\text {OFF }}$ ratio of $1.94 \times 10^{9}$. Furthermore, the impact of doping concentration, critical geometric parameters, gate work-function and bias are discussed, which benefits to adopt appropriate parameters to achieve excellent performance.

\section{Device Structure And Simulation Methodology}

3-D schematic view of conventional NT-TFET and proposed EHBNT-TFET are shown in Fig. 1(a) and (b), respectively, and the corresponding cross-sectional view along A-A' cut-plane are presented in Fig. 1(c) and (d). Conventional NT-TFET has a symmetric inner/outer-gate with the same metal work-function, and generally the same bias is applied to the inner/outer-gate. The EHBNT-TFET presents an intentional misaligned inner/outer-gate. The inner-gate has a higher work-function, compared to the outer-gate. When a positive voltage is applied to the outer-gate and a negative voltage is applied to the inner-gate, a biasinduced electron-hole bilayeris formed in the thin silicon nanotube channel. Consequently, an abrupt $\mathrm{p}^{+}-\mathrm{n}^{+}$ junction is formed in the intrinsic channel. Then the line tunneling occurs between electron-hole bilayer, i.e., the overlapped region between the inner-gate and outer-gate. Furthermore, the underlap at the source and drain end could eliminate parasitic tunneling current in OFF-state. Referring to the fabrication of previous NT-TFET [24-27], a possible process flow for proposed EHBNT-TFET is presented in Fig. 2. The main difference in EHBNT-TFET is the formation of misaligned inner/outer-gate using different materials.

The simulations are performed using Sentaurus TCAD [28]. Besides the regular physical models, dynamic nonlocal path band-to-band tunneling (BTBT) and density gradient (DG) model are also activated. Dynamic BTBT adopts Wentzel-Kramers-Brillouin (WKB) approach to account for phonon-assisted tunneling process [29]. DG model is used to consider the quantum confinement effect. For the moment, trap-assisted tunneling process has not been considered in our simulations. The simulation environment is calibrated with the previous result of conventional NT-TFET under the same geometric parameters [24]. As shown in Fig. 3(a), the simulated transfer curve shows a good agreement with the data in [24]. For tunneling occurring at electron-hole bilayer, the correct computation of carrier density is critical. Therefore, DG model has been calibrated on the solution of the Poisson-Schrödinger equation with the structure in Fig. 1(c). Calibrated DG model parameters are given as follows: $y_{n}=7, \gamma_{p}=10.5, \eta_{n}=1.02, \eta_{p}=0.8, \theta_{n}=$ $0.91, \theta_{p}=0.97$. As shown in Fig. $3(\mathrm{~b})$, charge distributions in the channel arising from calibrated density gradient model matches properly with Schrödinger results for EHBNT-TFET. 
The key physical parameters of simulated NT-TFET and EHBNT-TFET are listed in Table 1. The intrinsic Si is adopted as the channel. To keep the same geometry, gate length of NT-TFET is $40 \mathrm{~nm}$, while the overlap between inner/outer-gateof EHBNT-TFET is $20 \mathrm{~nm}$, with a $10 \mathrm{~nm}$ underlap between inner-gate (outer-gate) and drain (source). The work-function of NT-TFET is tuned to obtain the same $I_{O F F}$ with EHBNT-TFET. By default, the applied voltage $V_{D D}$ is $0.5 \mathrm{~V}$.

\section{Results And Discussion}

\subsection{Characteristics of NT-TFET and EHBNT-TFET}

Transfer characteristics for both NT-TFET and EHBNT- TFET are depicted in Fig. 4(a). For EHBNT-TFET, the outer-gate controls the switch between OFF-state and ON-state. Compared to the conventional NTTFET, EHBNT-TFET offers an increased $I_{O N}$ by 57.2 times without any $I_{O F F}$ degradation. Here, $I_{O F F}$ is defined as drain current when the outer-gate bias $\left(V_{O G}\right)$ is $0 \mathrm{~V}$ and inner-gate bias $\left(V_{I G}\right)$ is $-0.1 \mathrm{~V}$. $I_{O N}$ is defined as drain current when $V_{O G}$ is $V_{D D}$ with fixed $V_{I G}$ of $-0.1 \mathrm{~V}$. Besides, proposed EHBNT-TFET presents a significant improvement in terms of drain current and subthreshold slope (SS), exhibiting a sub-60 $\mathrm{mV} / \mathrm{dec} S S$ for seven orders of magnitude of drain current, as shown in Fig. 4(b). Furthermore, the minimum $S S$ in EHBNT-TFET is $2.1 \mathrm{mV} / \mathrm{dec}$, while it is $28.5 \mathrm{mV} / \mathrm{dec}$ in NT-TFET. The steep OFF/ON switch offers a reduced $S S$ which proves the excellent gate control in EHBNT-TFET.

In TFET devices, $I_{O N}$ is governed by the inter-band tunneling current across tunneling barrier. With WKB approximation, the tunneling current is calculated as [30]

$$
I_{B T B T} \propto A e^{\left(-\frac{4 \times \sqrt{2 m^{*}} \times E_{g}^{3 / 2}}{3 q \hbar E}\right)}
$$

1

Where $m^{*}, E_{g}, q$ and $\hbar$ is carrier effective mass, band gap, elementary charge and Planck's constant, respectively. $E$ is electric field across tunneling junction. $A$ is the tunneling area. The improved $I_{O N}$, in the proposed EHBNT-TFET can first be ascribed to the increased tunnel area $A$. In conventional NT-TFET, the point tunneling dominates and the tunneling area equals the interface between source and channel. However, the line tunneling exists in EHBNT-TFET and the tunneling area equals the overlap between inner-gate and outer-gate. As indicated in Fig. 4(c), EHBNT-TFET obtains a larger tunneling area and then an improved $I_{O N}$ is expected. Besides, the enhanced electric field also contributes to improved $I_{O N}$, as demonstrated in Fig. 4(d). The maximum E in NT-TFET is about $2.2 \mathrm{MV} / \mathrm{cm}$, while it is $2.7 \mathrm{MV} / \mathrm{cm}$ in EHBNT-TFET. Furthermore, the maximum $E$ in EHBNT-TFET appears around the inner/outer-gate overlap, and it is parallel to line tunneling direction. Thus, an improved BTBT rate exists in EHBNT-TFET.

To evaluate the physical mechanism further, the energy band diagrams of two transistors along B-B' (narallel to channel) and C.-C' (nernendicular to channel) cut-line are shown in Fig. 5(a) (d). For EHBNTLoading [MathJax]/jax/output/CommonHTML/jax.js 
TFET, there is no overlap between the conduction band and valence band of channel in OFF-state, no matter along channel or perpendicular to channel, as shown in Fig. 5(a) and (b). As $V_{O G}$ increases, the energy band bends downward and energy overlap appears. The tunneling distance along $C-C^{\prime}$ cut-line is much shorter than that along B-B' cut-line. Therefore, the line tunneling dominates in EHBNT-TFET, and the lateral point tunneling from source to channel is negligible. On the contrary, as demonstrated in Fig. 5(c) and (d), only point tunneling along the source/channel interface exits in NT-TFET. Due to the limited silicon channel thickness, the line tunneling distance in EHBNT-TFET is greatly lower than the point tunneling distance in NT-TFET, as presented in Fig. 5(e). Furthermore, the line tunneling distance decreases with $V_{O G}$ increasing. The total tunneling strength in both devices is depicted in Fig. 5(f) as a function of gate voltage. It is the smaller tunneling distance that results in the improvement of tunneling rate. The short tunneling distance and large tunneling rate contribute to the improved tunneling strength in EHBNT-TFET.

The dynamic performance of NT-TFET and EHBNT-TFET is compared in Table 2. The intrinsic delay time $\tau$ is calculated as $C_{g g} \times V_{D D} / I_{O N}$ [31], where $C_{g g}$ is the extracted gate capacitance. $C_{g g}$ of EHBNT-TFET is lower than that of NT-TFET by $59 \%$ due to the existence of underlap between inner-gate (outer-gate) and drain (source). Furthermore, intrinsic delay time $\tau$ of EHBNT-TFET is reduced about 142 times, due to the combined result of decreased $C_{g g}$ and improved $I_{O N}$.

The transfer characteristics of EHBNT-TFET under various supply voltage is depicted in Fig. 6(a). The extracted transition voltage $\left(V_{T R}\right)$ keeps constant while $I_{O N}$ declines as $V_{D S}$ decreases from $0.5 \mathrm{~V}$ to $0.1 \mathrm{~V}$, as shown in Fig. $6(\mathrm{~b}) . V_{T R}$ is defined as the outer-gate voltage where the line tunneling starts, and $S S$ has the minimum value [17]. As the outer-gate voltage increases to $V_{T R}$ electrons start to tunnel from the valence band of induced $\mathrm{p}^{+}$layer to the conduction band of induced $\mathrm{n}^{+}$layer in channel. Here the line tunneling in EHBNT-TFET is dominated by gate electric field, while is not affected by drain voltage. Therefore, a constant $V_{T R}$ is expected as $V_{D S}$ increases. A lower $V_{T R}$ could allow a lower supply voltage.

\subsection{Impact of doping in EHBNT-TFET}

The impact of drain doping $\left(N_{D}\right)$ on EHBNT-TFET under various underlap between inner-gate and drain $\left(L_{\text {IGUD }}\right)$ is depicted in Fig. 7, where source doping $\left(N_{S}\right)$ is fixed at $5 \times 10^{19} \mathrm{~cm}^{-3}$ and underlap between outergate and source ( $L_{\text {OGUS }}$ ) is fixed at $10 \mathrm{~nm}$. $I_{\text {OFF }}$ remains nearly unchanged for lower $N_{D}$, while it begins to increase once $N_{D}$ exceeds $1 \times 10^{18} \mathrm{~cm}^{-3}$, and the increasing trend is weakened as $L_{\text {IGUD }}$ increases, as depicted in Fig. 7(a). $I_{O N}$ monotonically increases as $N_{D}$ increases for a small $L_{I G U D}$, while it keeps nearly unchanged once $L_{\text {IGUD }}$ exceeds $6 \mathrm{~nm}$, as depicted Fig. $7\left(\right.$ b). Furthermore, a larger $L_{\text {IGUD }}$ presents an improved $I_{O N}$. In EHBNT-TFET, $I_{O F F}$ is comprised of SRH recombination as well as parasitic tunneling from drain to induced $\mathrm{p}^{+}$layer.As $N_{D}$ increases, the carrier lifetime declines and then $\mathrm{SRH}$ recombination increases. As shown in Fig. 8(a), SRH recombination dominates $I_{O F F}$ under lower $N_{D}$, and the parasitic tunneling dominates $I_{O F F}$ under high $N_{D}$. The sharply increased $I_{\text {OFF }}$ under $N_{D}$ of $1 \times 10^{19} \mathrm{~cm}^{-3}$ is the result of Loading [MathJax]/jax/output/CommonHTML/jax.js ed in Fig. 8(b), there is no band overlap between drain and 
channel under low $N_{D}$. However, when $N_{D}$ rises to $1 \times 10^{19} \mathrm{~cm}^{-3}$, the energy band has overlap and the tunneling distance between drain and channel is enough thin, leading to an increased parasitic tunneling current. As shown in Fig. 8(c), the tunneling distance between drain/channel junction increases under larger $L_{\text {IGUD }}$. Thus, $N_{D}$ has less impact on $I_{O F F}$ with $L_{I G U D}$ increasing. The trend of $I_{O N}$ could be illustrated by Fig. 8(d), taking the case of $L_{I G U D}=2 \mathrm{~nm}$ as an example. For a smaller $N_{D}$, the high barrier between channel and drain leads to a low $I_{O N}$. With $N_{D}$ increasing, the energy band in drainbends downward, resulting a lower barrier and then a higher $I_{O N}$. The effect of larger $L_{I G U D}$ is similar to that of high $N_{D}$. Furthermore, the impact of $N_{D}$ on barrier is weakened for a larger $L_{I G U D}$, so that $I_{O N}$ is barely affected. It is observed that the proposed device achieves better dc performance at $L_{\text {IGUD }}=10 \mathrm{~nm}$ and $N_{D}=1 \times 10^{18}$ $\mathrm{cm}^{-3}$.

Fig. 9 depicts the impact of $N_{S}$ under various $L_{\text {OGUS }}$ where $N_{D}$ is fixed at $1 \times 10^{18} \mathrm{~cm}^{-3}$ and $L_{\text {IGUD }}$ is fixed at $10 \mathrm{~nm}$. As shown in Fig. 9(a). When $N_{S}$ is lower than $1 \times 10^{19} \mathrm{~cm}^{-3}, I_{O F F}$ remains unchanged for arbitrary $L_{\text {OGUS }}$. As $N_{S}$ further increase, $I_{\text {OFF }}$ gradually increase especially for the smaller $L_{\text {OGUS }}$ such as $2 \mathrm{~nm}$ and 4 nm. $I_{O N}$ increases with $N_{S}$ increasing especially forsmaller $L_{O G U S}$ as shown in Fig. 9(b). Here the parasitic tunneling between drain and channel could be ignored due to the large $L_{\text {IGUD }}$ and low $N_{D}$. As shown in Fig. 10(a), SRH recombination keeps constant and therefore BTBT from source to channel dominates the trend with $N_{S}$. As $N_{S}$ rises up to $5 \times 10^{19} \mathrm{~cm}^{-3}$, particularly for $L_{\text {OGUS }}=2 \mathrm{~nm}$, electron begins to tunnel from source to channel. The larger $I_{\text {OFF }}$ under $N_{S}$ of $1 \times 10^{20} \mathrm{~cm}^{-3}$ is the result of energy band overlap between source and channel in OFF-state. Due to low work-function of the outer-gate, the increased $N_{S}$ leads to a declined tunneling distance and an improved tunneling rate from source to channel under low $L_{O G U S}$, as represented in Fig. 10(b). Thus, the parasitic tunneling between source and channel increases and dominants $I_{O F F}$ under low $L_{\text {OGUS }}$ and high $N_{S}$ leading to a large $I_{\text {OFF. }}$. However, with $L_{\text {OGUS }}$ increasing, tunneling between source and channel hardly takes place due to large tunneling distance. The increased trend of $I_{O N}$ is because high $N_{S}$ could provide more hole in induced $\mathrm{p}^{+}$layer. Similarly, the impact of $N_{S}$ is weakened by larger $L_{\text {OGUS }}$. Considering the tradeoff between $I_{O F F}$ and $I_{O N}, L_{O G U S}=10 \mathrm{~nm}$ is recommended as the most suitable geometry. Meanwhile, lower $N_{S}$ could be adopted and the requirement of extremely steep doping profiles between source and channel is not necessary for EHBNT-TFET.

In conventional TFET $I_{O F F}$ is mainly affected by $N_{D}$ and $I_{O N}$ is only sensitive to $N_{S}$ [31]. However, in EHBNTTFET $I_{O N}$ is dominated by the line tunneling in channel, and the large underlap at source and drain end eliminate the effect of $N_{D} / N_{S}$ on $I_{O F F}$. Therefore, $N_{D}$ and $N_{S}$ have little impact on device performance of EHBNT-TFET with large $L_{I G U D}$ and $L_{O G U S}$.

\subsection{Impact of geometric parameters in EHBNT-TFET}

The effect of overlap between inner/outer-gate $\left(L_{I O G O}\right)$ and inner-gate diameter $\left(D_{I G}\right)$ are shown in Fig. 11. $I_{O N}$ and $I_{O N} / I_{O F F}$ gradually improves with $L_{I O G O}$ and $D_{I G}$ increasing, due to the increased line tunneling Loading [MathJax]/jax/output/CommonHTML/jax.js Iso leads to a degraded $I_{O F F} I_{O N}$ has greater improvement 
than $I_{O F F}$ so that $I_{O N} / I_{O F F}$ is improved. The EHBNT-TFET provides much higher scalability with the help of circular cylinder channel. Compared with EHBTFET, tunneling area can be extended with smaller device size by larger $D_{I G}$ for EHBNT-TFET. Larger $L_{1 O G O}$ can achieve higher current drivability without any integration density loss. Furthermore, the proposed EHBNT-TFET shows much higher normalized $I_{O N}$ and $I_{\text {ON }} / I_{\text {OFF }}$ than conventional EHBTFET due to better gate control, as shown in Table 3.

As shown in Fig. 13, the extracted $V_{T R}$ decreases, i.e., the line tunneling starts at lower $V_{O G}$ with $T_{S C}$ increasing. The energy band perpendicular to channel in OFF-state is presented in Fig. 13(b). No overlap exists between the conduction band and valence band when $T_{S C}$ increases from $3.5 \mathrm{~nm}$ to $5.5 \mathrm{~nm}$. The conduction band near outer-gate is $0.189 \mathrm{eV}$ higher than the valence band near inner-gate at $T_{S C}=3.5$ $\mathrm{nm}$, while it is only $0.011 \mathrm{eV}$ at $T_{S C}=5.5 \mathrm{~nm}$. Therefore, a larger $V_{O G}$ isrequired at $T_{S C}=3.5 \mathrm{~nm}$ to pull down the energy band to create the line tunneling in channel, compared to the case of $T_{S C}=5.5 \mathrm{~nm}$. As a result, a higher $V_{T R}$ is obtained for smaller $T_{S C}$. According to the analysis above, a $T_{S C}$ of $4 \mathrm{~nm}$ could be adopted to improve dc performance of EHBNT-TFET.

\subsection{Impact of gate work-function and inner-gate bias in EHBNT-TFET}

The effect of work-function of outer-gate $\left(\Phi_{O G}\right)$ is shown in Fig. 14. $I_{O F F}$ and $I_{O N}$ increases monotonously, while $V_{T R}$ decreases as $\Phi_{O G}$ decreases from $3.95 \mathrm{eV}$ to $3.75 \mathrm{eV}$. The lower $\Phi_{O G}$ causes more inverse electron in the channel, leading to a higher $S R H$ recombination rate and an increased $I_{O F F}$. As $\Phi_{O G}$ decreases, the energy band of channel beneath outer-gate moves downward and a steeper $\mathrm{p}^{+}-\mathrm{n}^{+}$junction is formed, as shown in Fig. 14(c). As a result, an increased $I_{O N}$ is observed due to the decreased tunneling distance and increased overlapped energy range. To explore the variation of $V_{T R}$ the energy band perpendicular to channel in OFF-state is plotted in Fig. 14(d). In the case of $\Phi_{O G}=3.95 \mathrm{eV}$, the conduction band near outer-gate is $0.138 \mathrm{eV}$ higher than the valence band near inner-gate, while it is only $0.01 \mathrm{eV}$ at $\Phi_{O G}=3.75 \mathrm{eV}$. As a result, a higher $\Phi_{O G}$ results in a larger $V_{T R}$.

The impact of work-function of inner-gate $\left(\Phi_{I G}\right)$ is shown in Fig. 15. In contrast to the case of $\Phi_{O G} I_{O F F}$ and $I_{O N}$ increases monotonously, while $V_{T R}$ decreases as $\Phi_{I G}$ increases from $5.25 \mathrm{eV}$ to $5.45 \mathrm{eV}$. The increased $\Phi_{I G}$ have the similar effect on device performance with decreased $\Phi_{O G}$. More carrier in the channel due to increased $\Phi_{I G}$ leads to an increased $I_{O F F}$. Similarly, by decreasing surface potential of the channel near inner-gate, the energy band of channel beneath inner-gate is pulled upward, as shown in Fig. 15 (c), resulting in an increased $I_{O N}$. As for transition voltage $V_{T R}$ the energy band perpendicular to channel in OFF-state is plotted in Fig. 15(d). In the case of $\Phi_{I G}=5.25 \mathrm{eV}$, the conduction band near outergate is $0.136 \mathrm{eV}$ higher than the valence band near inner-gate, while it is only $0.013 \mathrm{eV}$ at $\Phi_{I G}=5.45 \mathrm{eV}$, leading to a lower $V_{T R}$ at high $\Phi_{I G}$. In short, thecharacteristics improve with increasing $\Phi_{I G}$. 
The effect of $\Phi_{I G}$ and $V_{I G}$ on EHBNT-TFET is compared by design of experiment in Table 4 . It is found that transfer characteristics of experiment No. 1 is exactly the same with that of experiment No. 3, experiment No. 2 and No. 4 is also the same. In short, the reduction of $\Phi_{I G}$ have the same effect on device performance with increased $V_{I G}$. The same effect of $\Phi_{I G}$ and $V_{I G}$ makes it more flexible for device and circuit design.

\section{Conclusion}

In this paper, a novel NT-TFET with an intentional misaligned inner/outer-gate has been proposed, achieving excellent static and dynamic performance. Line tunneling between electron-hole bilayer offers the steep transition from OFF-state to $O N$-state and high drive current. $I_{O N}$ is found to improve by 57.2 times, and a sub- $60 \mathrm{mV} / \mathrm{dec}$ subthreshold slope for seven orders of magnitude of drain current is obtained. In addition, the impact of doping on static performance was discussed in detail. The performance of EHBNT-TFET is almost unaffected by source and drain doping with large underlap. The immunity of doping eliminates the requirement for formation of abrupt source/channel junction and high thermal budget in fabrication process. Meanwhile, the characteristics of various geometric parameters was studied to obtain better device performance. Due to the electron-hole bilayer induced by appropriate gate configuration, gate work-function and bias are significantly important to offer superior performance.

\section{Declarations}

Acknowledgments The authors are grateful to National Natural Science Foundation of China, Natural Science Foundation of Shang under Grant, Shanghai Science and Technology Innovation Action Plan and Science and Technology Commission of Shanghai Municipality for the financial support.

Author contributions Conceptualization, methodology, writing- original draft preparation: Xueke Wang; Data analysis: Xueke Wang and Yabin Sun; Writing- review and editing: Yun Liu and Xiaojin Li; Supervision: Ziyu Liu and Yanling Shi; Resources and funding acquisition: Ziyu Liu.

Funding This work was supported in part by National Natural Science Foundation of China under Grant 61974056, in part by Natural Science Foundation of Shang under Grant 19ZR1471300, in part by Shanghai Science and Technology Innovation Action Plan under Grant 19511131900, and in part by Science and Technology Commission of Shanghai Municipality under Grant 14DZ2260800.

Availability of data and material The data and material that support the findings of this study are available on request from the corresponding author.

Code availability Not Applicable.

Competing interest Not Applicable. 
Consent to Participate Not applicable.

Consent for Publication Yes.

Compliance with Ethical Standards

Disclosure of potential conflicts of interest Authors have no conflicts of interest to declare that are relevant to the content of this article.

Research involving Human Participants and/or Animals Not applicable.

Informed consent Not applicable.

\section{References}

1. Taur Y (2002) CMOS design near the limit of scaling. IBM J Res Dev 46:213-222

2. Ionescu AM, De Michielis L, Dagtekin N, et al (2011) Ultra low power: Emerging devices and their benefits for integrated circuits. Tech Dig - Int Electron Devices Meet IEDM 378-381

3. Woo Young C, Byung-Gook P, Jong Duk L, Tsu-Jae King L (2007) Tunneling Field-Effect Transistors (TFETs) With Subthreshold Swing (SS) Less Than $60 \mathrm{mV} / \mathrm{dec}$. IEEE Electron Device Lett 28:743-745

4. Ionescu AM, Riel H (2011) Tunnel field-effect transistors as energy-efficient electronic switches. Nature 479:329-337

5. Khan Al, Yeung CW, Hu C, Salahuddin S (2011) Ferroelectric negative capacitance MOSFET:

Capacitance tuning \& antiferroelectric operation. Tech Dig - Int Electron Devices Meet IEDM 255-258

6. Gopalakrishnan K, Griffin PB, Plummer JD (2005) Impact ionization MOS (I-MOS)-Part I: Device and circuit simulations. IEEE Trans Electron Devices 52:69-76

7. Theis TN, Solomon PM (2010) It's Time to Reinvent the Transistor! Science 1600-1601

8. Raushan MA, Alam N, Siddiqui MJ (2018) Dopingless Tunnel Field-Effect Transistor with Oversized Back Gate: Proposal and Investigation. IEEE Trans Electron Devices 65:4701-4708

9. Kumar MJ, Member S, Janardhanan S (2013) Doping-less Tunnel Field Effect Transistor: Design and Investigation. IEEE Trans Electron Devices 60:3285-3290

10. Raad BR, Tirkey S, Sharma D, Kondekar P (2017) A New Design Approach of Dopingless Tunnel FET for Enhancement of Device Characteristics. IEEE Trans Electron Devices 64:1830-1836

11. Hanna AN, Fahad HM, Hussain MM (2015) InAs/Si hetero-junction nanotube tunnel transistors. Sci 
12. Kim SH, Agarwal S, Jacobson ZA, et al (2010) Tunnel field effect transistor with raised germanium source. IEEE Electron Device Lett 31:1107-1109

13. Boucart K, Ionescu AM (2007) Double-Gate Tunnel FET With High-K Gate Dielectric. IEEE Trans Electron Devices 54:1725-1733

14. Bhuwalka KK, Schulze J, Eisele I (2004) Performance enhancement of vertical tunnel field-effect transistor with SiGe in the $\delta p+$ layer. Japanese J Appl Physics 43:4073-4078

15. Abdi DB, Kumar MJ (2014) In-built N+ Pocket p-n-p-n tunnel field-effect transistor. IEEE Electron Device Lett 35:1170-1172

16. Seo JH, Yoon YJ, Lee S, et al (2015) Design and analysis of Si-based arch-shaped gate-all-around (GAA) tunneling field-effect transistor (TFET). Curr Appl Phys 15:208-212

17. Lattanzio L, De Michielis L, Ionescu AM (2012) The electron-hole bilayer tunnel FET. Solid State Electron 74:85-90

18. Lattanzio L, Michielis L De, Member S, et al (2012) Complementary Germanium Electron-Hole Bilayer Tunnel FET for Sub-0.5-V Operation. IEEE Electron Device Lett 33:167-169

19. Padilla JL, Alper C, Godoy A, et al (2015) Impact of Asymmetric Configurations on the Heterogate Germanium Electron-Hole Bilayer Tunnel FET Including Quantum Confinement. IEEE Trans Electron Devices 62:3560-3566

20. Kim S, Choi WY, Park BG (2018) Vertical-structured electron-hole bilayer tunnel field-effect transistor for extremely low-power operation with high scalability. IEEE Trans Electron Devices 65:2010-2015

21. Fahad HM, Smith CE, Rojas JP, Hussain MM (2011) Silicon nanotube field effect transistor with coreshell gate stacks for enhanced high-performance operation and area scaling benefits. Nano Lett 11:4393-4399

22. Hanna AN, Hussain MM (2015) Si/Ge hetero-structure nanotube tunnel field effect transistor. J Appl Phys 117:014310

23. Shell C, Tfet DG, Si W, et al (2020) Design and Performance Optimization of Novel Core-Shell Dopingless GAA-Nanotube TFET With $\mathrm{Si}_{0.5} \mathrm{Ge}_{0.5}$-Based Source. IEEE Trans Electron Devices 67:789-795

24. Fahad HM, Hussain MM (2013) High-performance silicon nanotube tunneling FET for ultralow-power logic applications. IEEE Trans Electron Devices 60:1034-1039

25. Tekleab D, Tran H H, Sleight J W, Chidambarrao D (2012) silicon nanotube mosfet. 
26. Musalgaonkar G, Sahay S, Saxena RS, Kumar MJ (2019) A line tunneling field-effect transistor based on misaligned core-shell gate architecture in emerging nanotube fets. IEEE Trans Electron Devices $66: 2809-2816$

27. Musalgaonkar G, Sahay S, Saxena RS, Kumar MJ (2019) Nanotube Tunneling FET with a Core Source for Ultrasteep Subthreshold Swing: A Simulation Study. IEEE Trans Electron Devices 66:44254432

28. Sentaurus TCAD User's Manual, Synopsys, Inc. (2018) Mountain View, CA, USA

29. Hurkx GAM, Klaassen DBM, Knuvers MPG (1992) A New Recombination Model for Device Simulation Including Tunneling. IEEE Trans Electron Devices 39:331-338

30. Boucart K (2010) Simulation of Double-Gate Silicon Tunnel FETs with a High-k Gate Dielectric. Ph.D. dissertation, École Polytechnoique Fédérale de Lausanne, Switzerland

31. Lee JS, Seo JH, Cho S, et al (2013) Simulation study on effect of drain underlap in gate-all-around tunneling field-effect transistors. Curr Appl Phys 13:1143-1149

\section{Tables}

Table 1 : Device parameters for the simulated NT-TFET and EHBNT-TFET

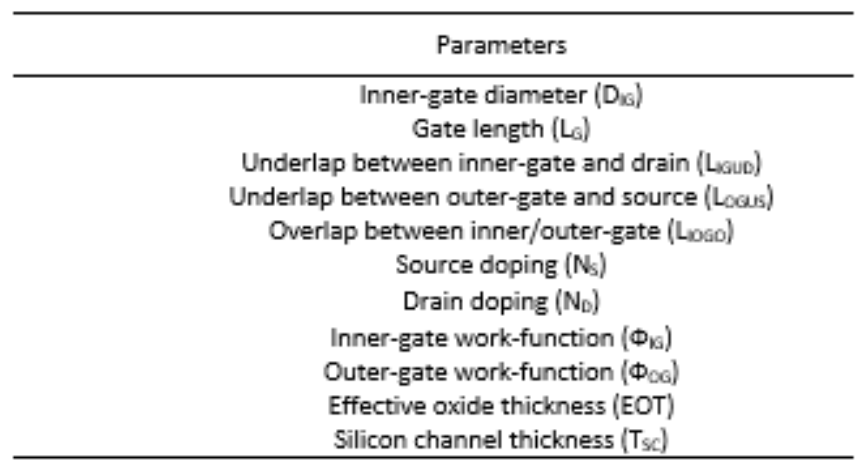

Table 2: Dynamic performance for the NT-TFET and EHBNT-TFET

\begin{tabular}{c}
\hline Parameters \\
\hline Gate capacitance $\left(\mathcal{C}_{\mathrm{m}}\right)$ \\
Intrinsic delay time $(\tau)$
\end{tabular}

Table 3 : Performance comparison of EHBTFET and EHBNT-TFET

\begin{tabular}{cc}
\hline Parameters \\
\hline $\operatorname{Loso}(\mathrm{nm})$ \\
$\operatorname{loff}(\mu \mathrm{A} / \mathrm{um})$ \\
$\operatorname{low}(\mu \mathrm{A} / \mathrm{um})$ \\
$\operatorname{lom} / /_{\mathrm{Lff}}$ \\
$\mathrm{V}_{\mathrm{oD}}(\mathrm{V})$ \\
\hline \hline Loading [MathJax]/jax/output/CommonHTML/jax.js
\end{tabular}


Table 4 : Experiments with different inner-gate work-function and bias for EHBNT-TFET

\begin{tabular}{cc}
\hline Number & $\Phi_{\mathrm{IG}}$ \\
\hline 1 & 5. \\
2 & 5. \\
3 & 5. \\
4 & 5. \\
\hline
\end{tabular}

\section{Figures}

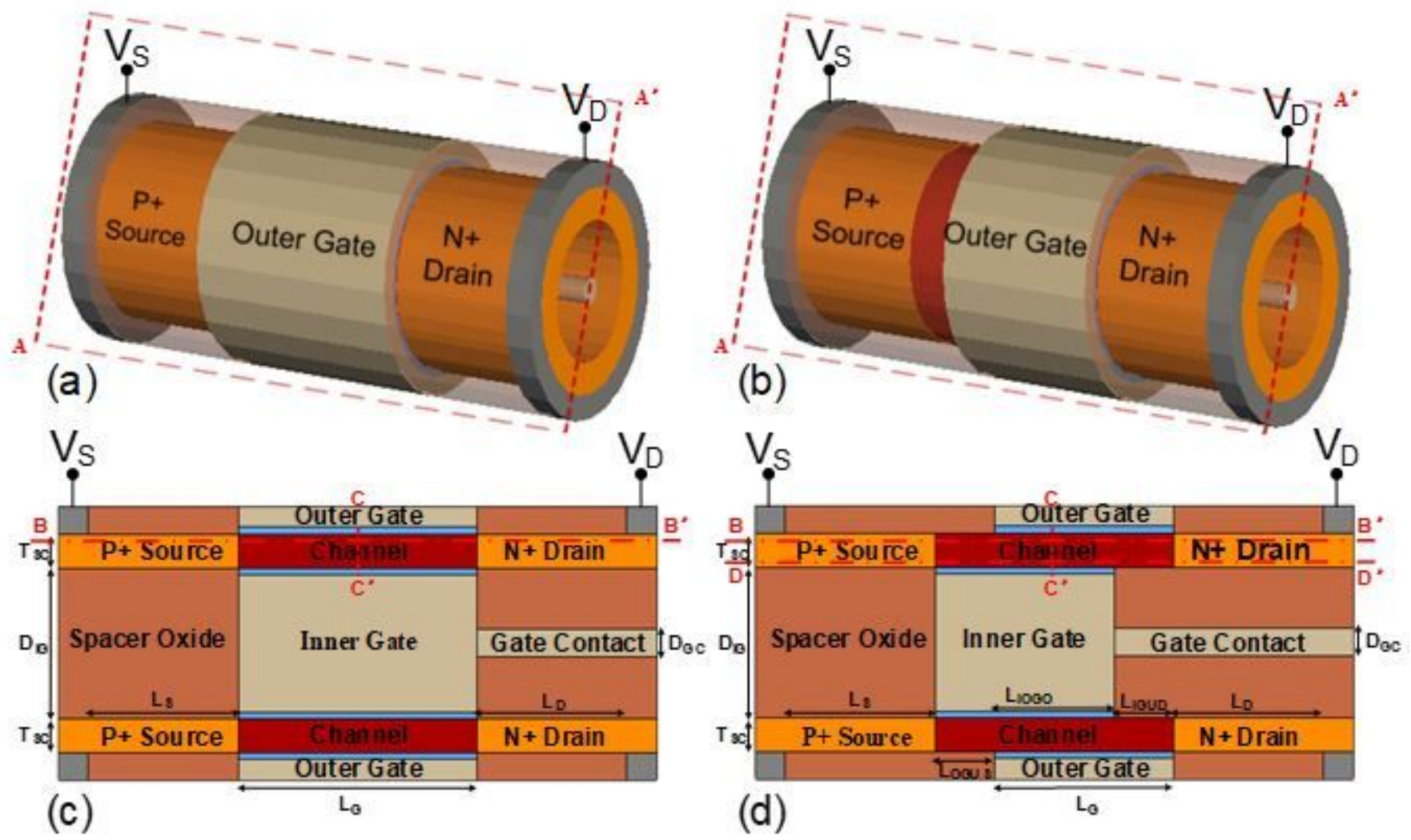

\section{Figure 1}

3-D schematic view of (a) conventional NT-TFET and (b) proposed EHBNT-TFET. The cross sectional view along A-A' cut-plane of (c) conventional NT-TFET and (d) proposed EHBNT-TFET. 


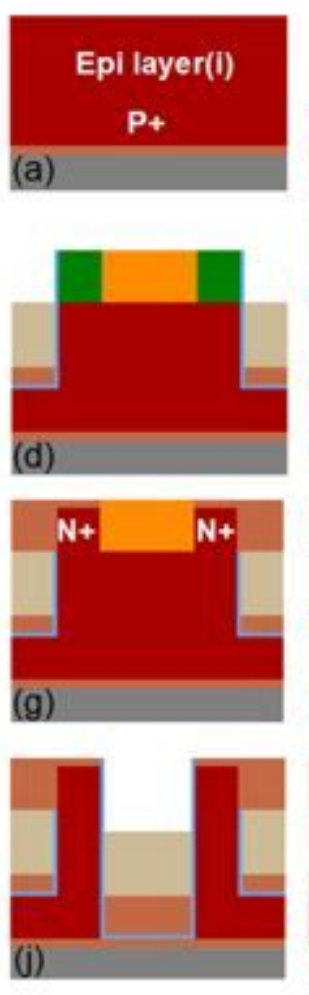

Back Silicon Sacrificial oxide
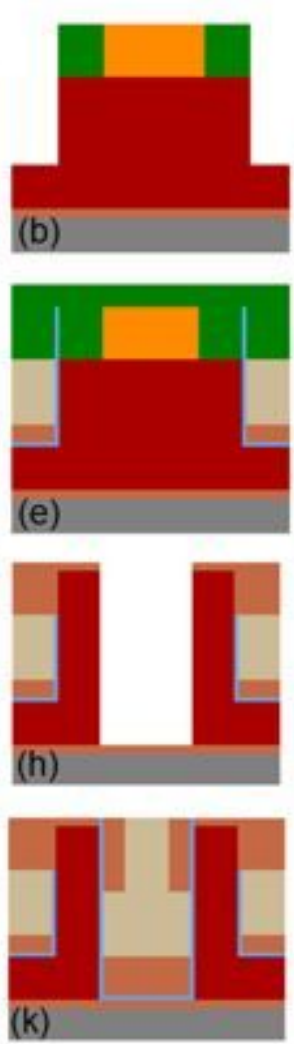

$\mathrm{SiO}_{2}$

Gate Metal
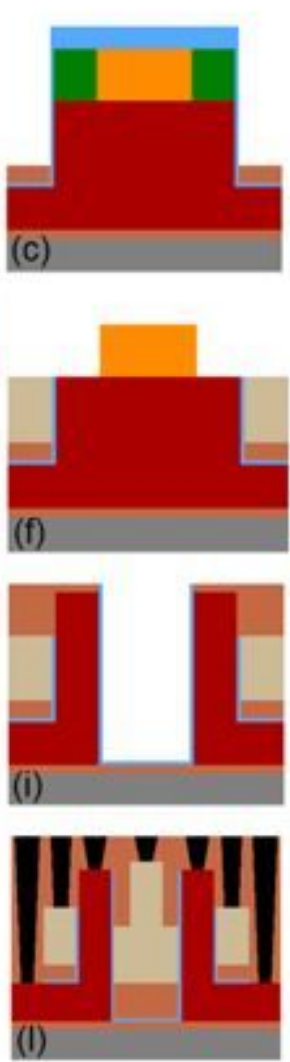

Active Silicon layer Silicon Nitride (a) SOI wafer \& Si epitaxy growth

(b) Cylindrical-shaped Si layer patterning

(c) Outer gateoxide \& spacer deposition

(d) Outer-gate deposition \& removal

(e) Sacrificial layer deposition \& planarization Selective etching

Si epitaxy growth \& spacer deposition

Trench creation

Inner gateoxide deposition

Spacer \& inner-gate deposition

(k) Spacer \& inner-gate contact deposition

(I) Source/Drain \& gate contact patterning

Dielectric

Contact

Figure 2

Possible fabrication process flow for EHBNT-TFET.

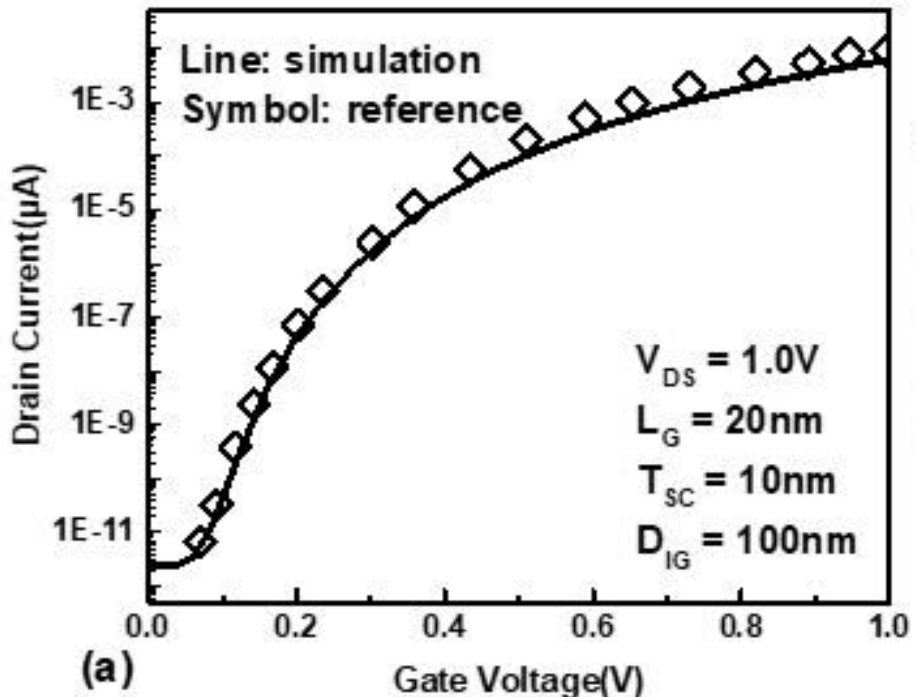

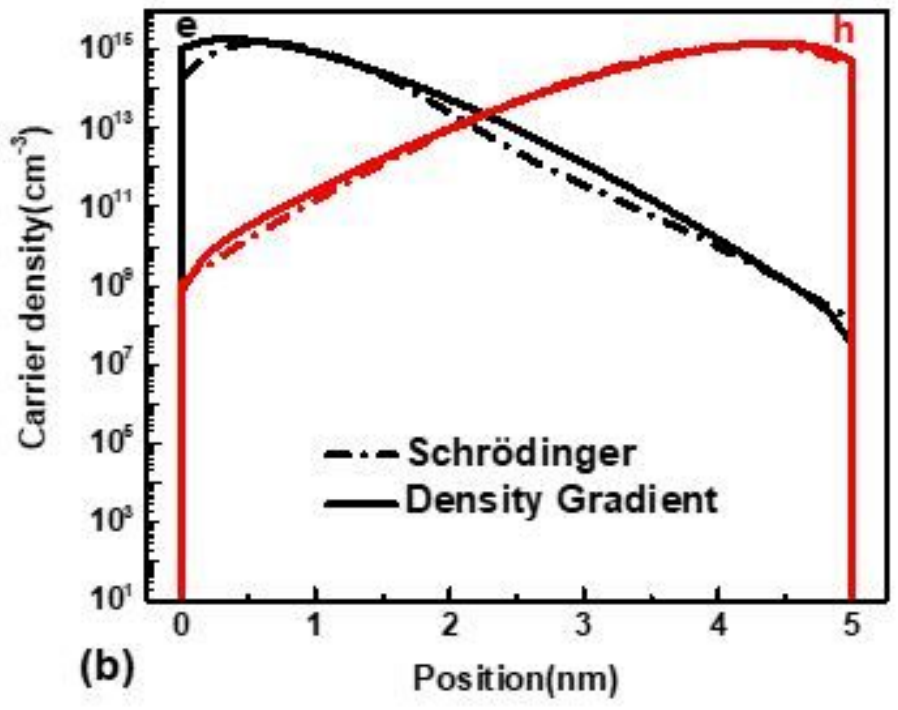

(b)
Position(nm)

Figure 3 
(a) Validation of the simulation environments by reproducing the transfer characteristic curve of [24]. (b) Carrier density distributions along $\mathrm{C}-\mathrm{C}^{\prime}$ cut-line arising from calibrated density gradient model and Schrödinger results for EHBNT-TFET.
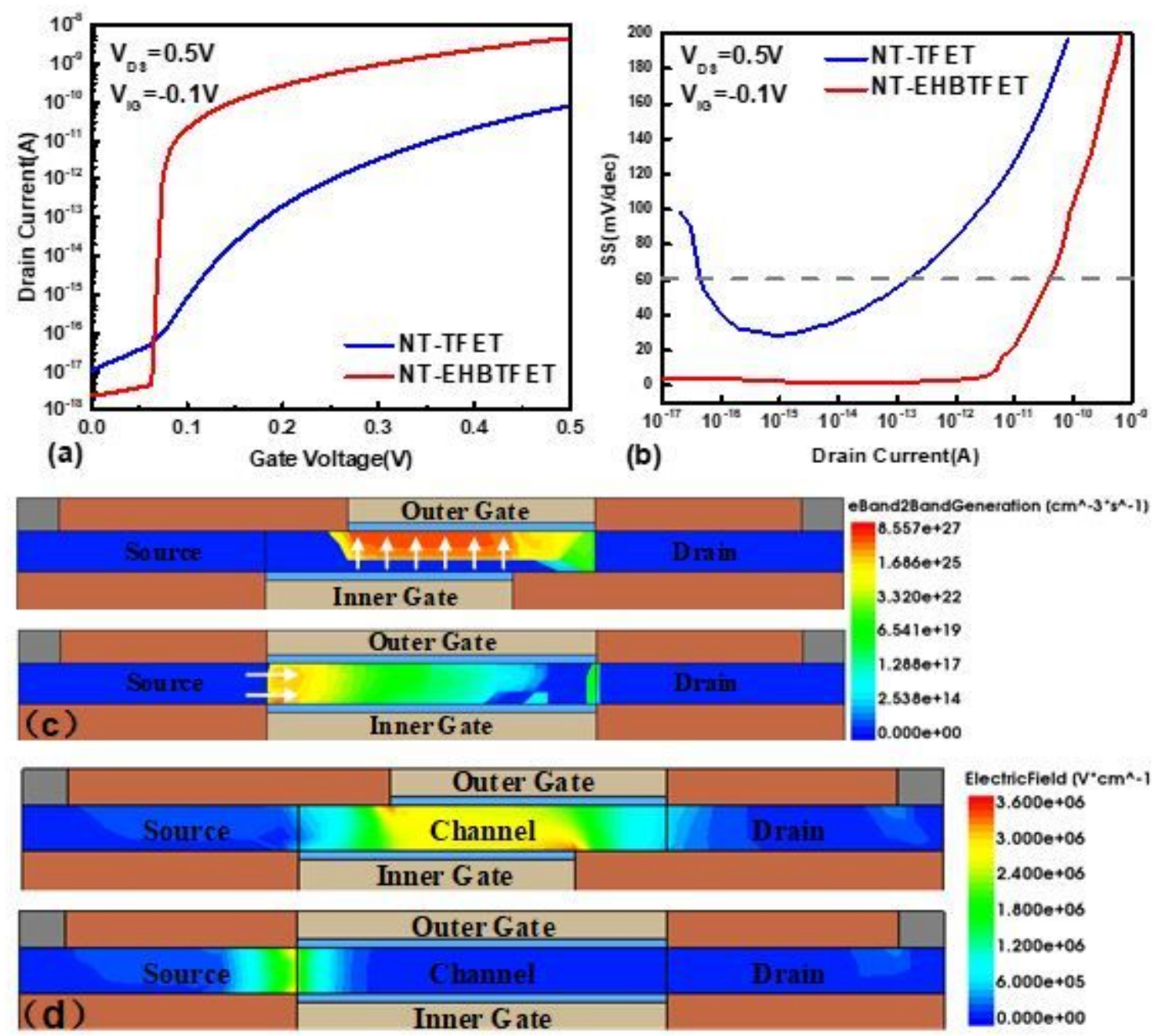

Electricfield $\left(V^{*} \mathrm{~cm}^{\wedge}-1\right.$

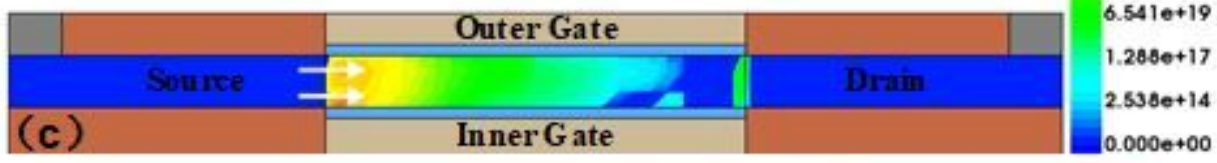

\section{Figure 4}

Comparison of (a) transfer curve and (b) SS of NT-TFET and EHBNT-TFET. (c) Electron BTBT generation profiles and (d) electric field distribution under $\mathrm{ON}$-state for both devices. 

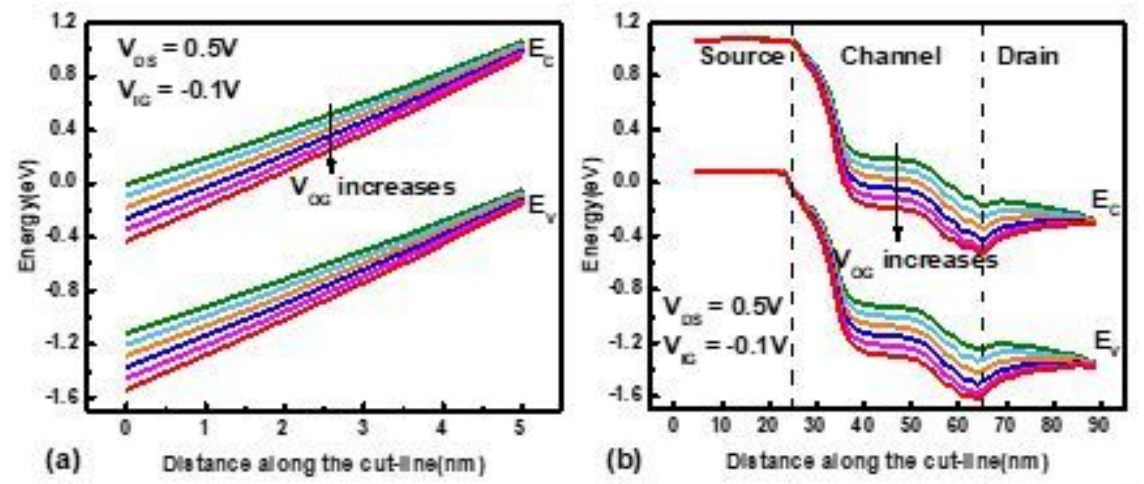

(a)
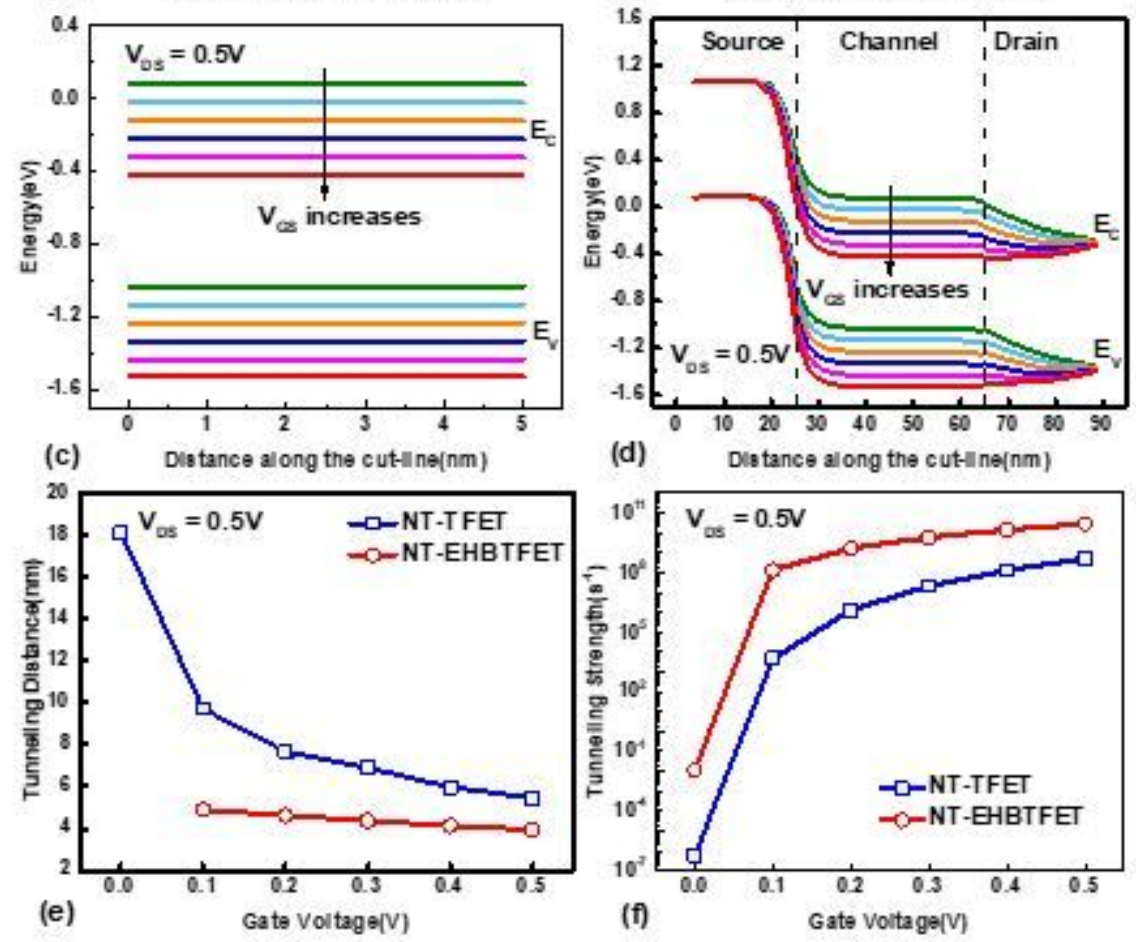

Figure 5

Variation of energy band for gate voltage from 0 to $0.5 \mathrm{~V}$ with step size of $0.1 \mathrm{~V}$ : EHBNT-TFET along (a) C$\mathrm{C}^{\prime}$ and (b) B-B' cut-line; NT-TFET along (c) C-C' and (d) B-B' cut-line. (e) Tunneling distance and (f) tunneling strength as function of gate voltage for both devices. 

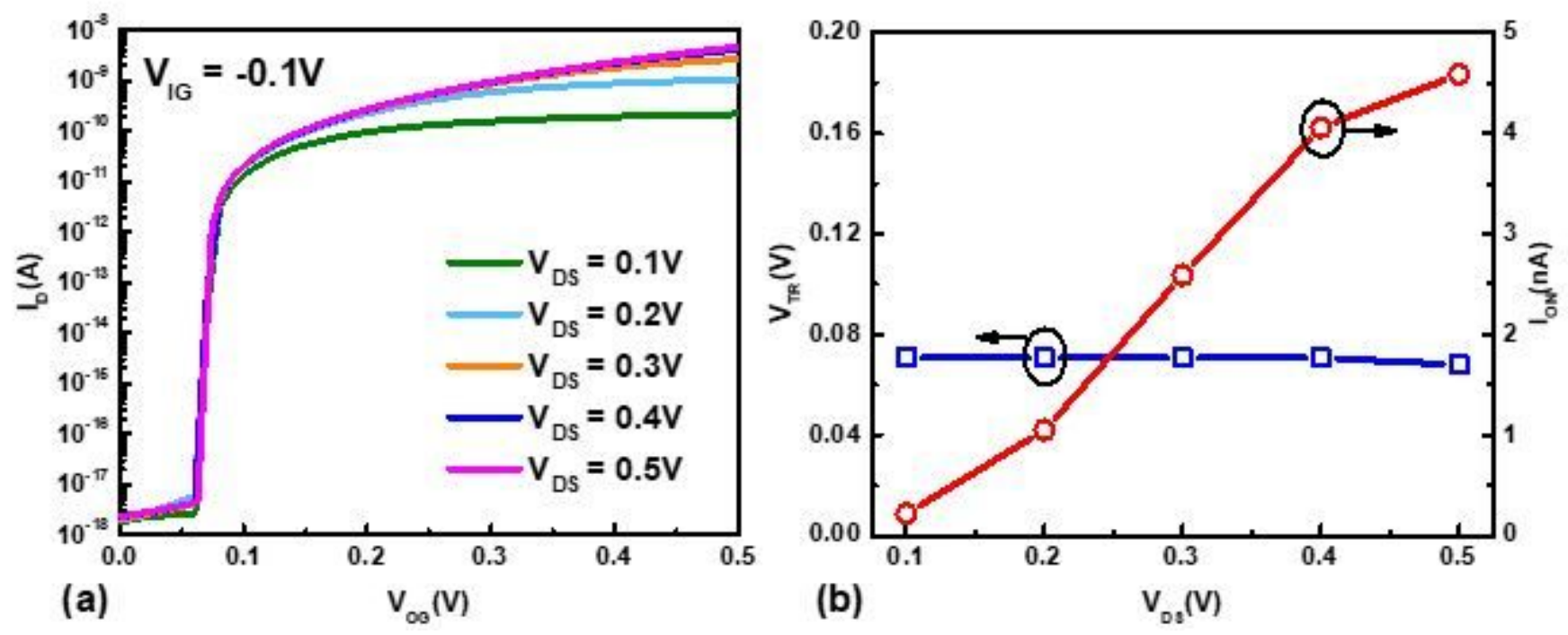

Figure 6

Transfer curve and (b) VTR and ION with various VDS for EHBNT-TFET.
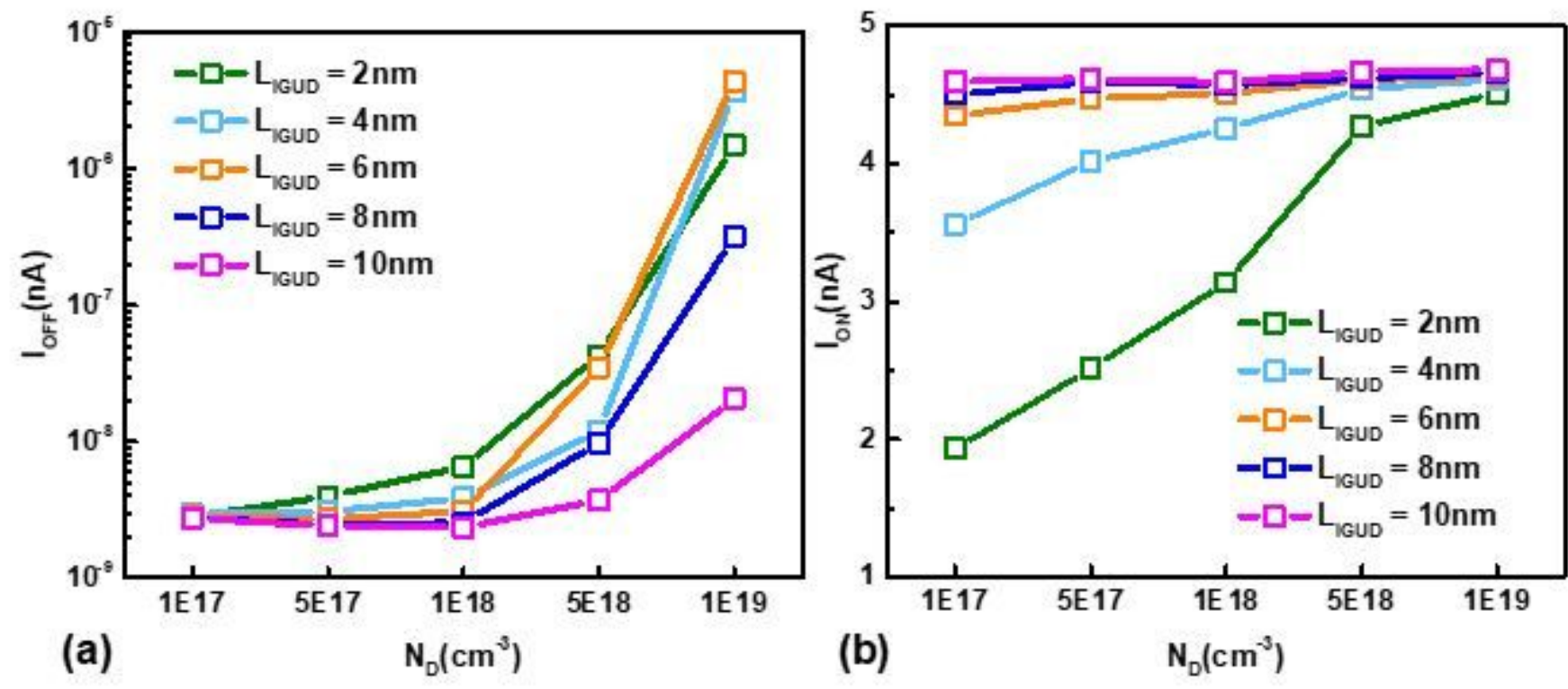

Figure 7

Impact of ND with various LIGUD on (a) IOFF and (b) ION for EHBNT-TFET. 


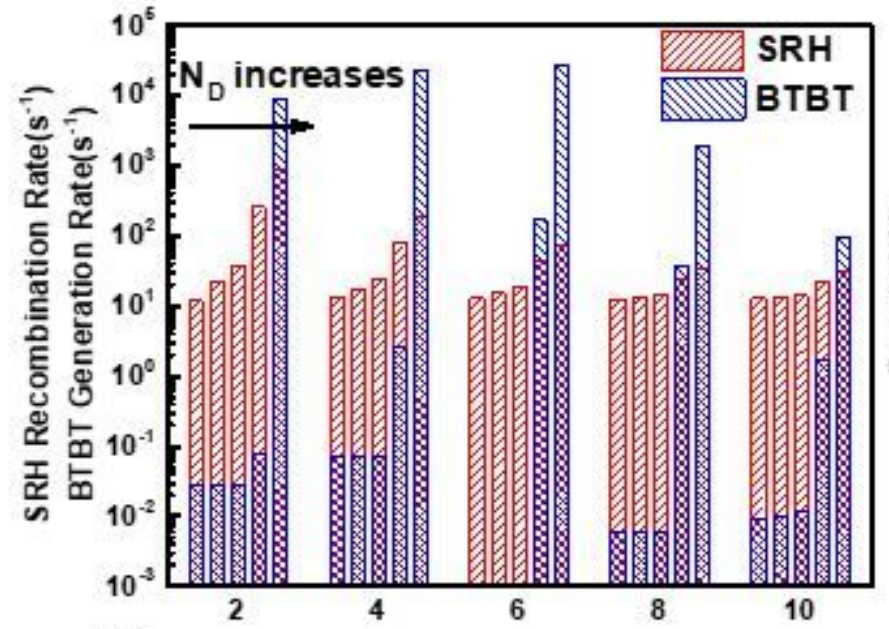

(a)

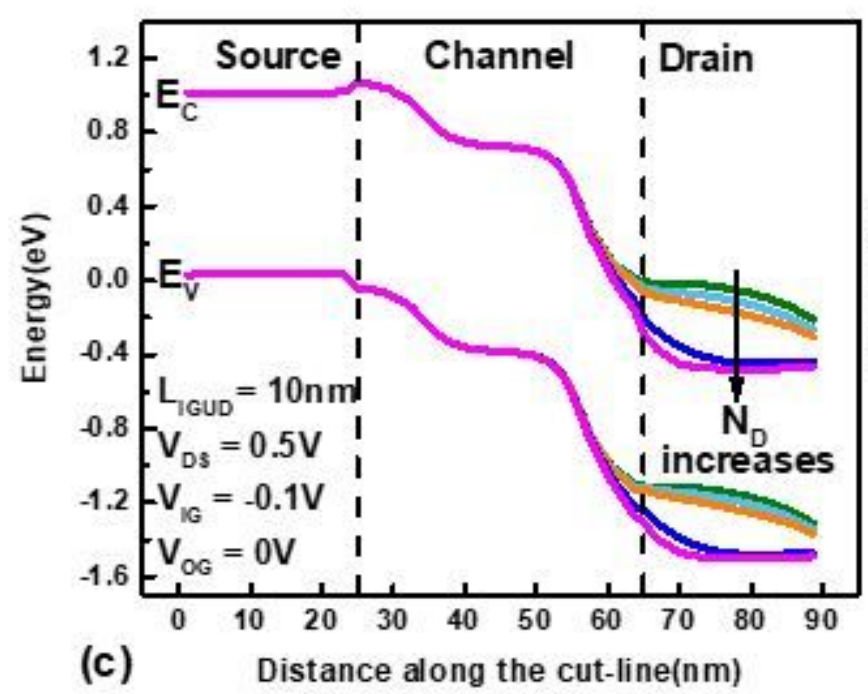

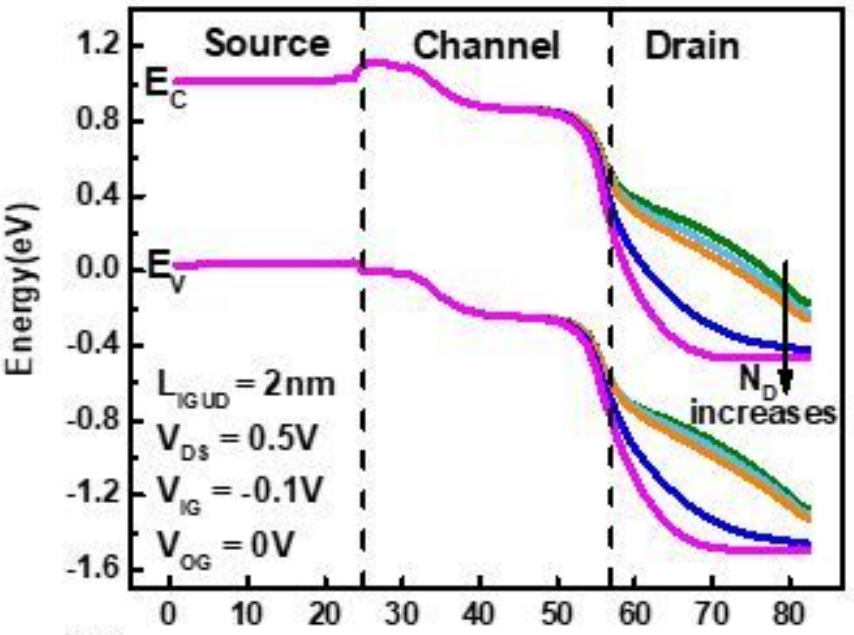

(b)

Distance along the cut-line(nm)

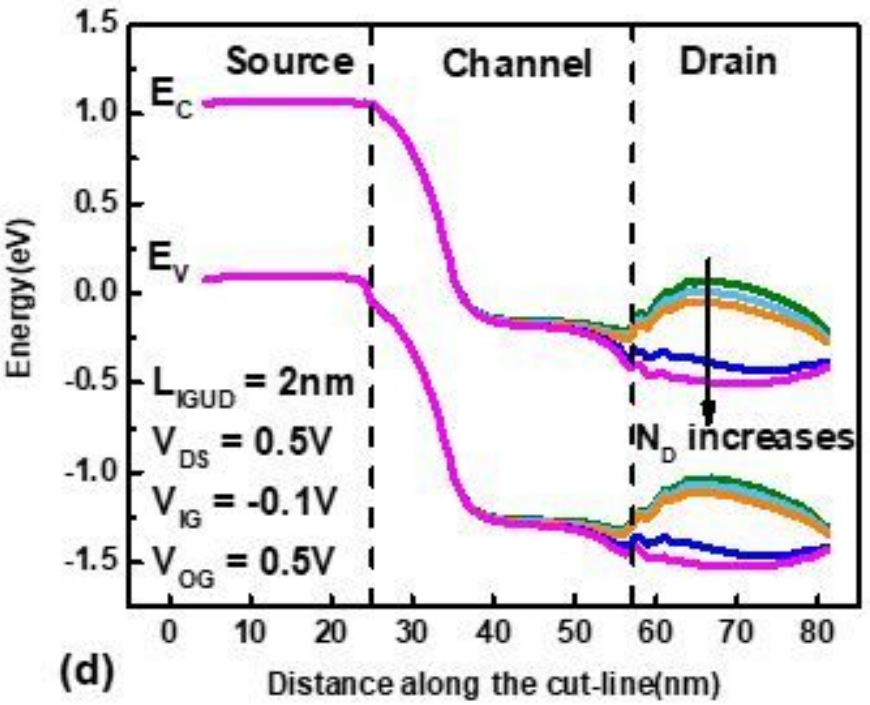

Figure 8

For EHBNT-TFET: (a) SRH recombination rate and BTBT generation rate under OFF-state with various LIGUD; Energy band along D-D' cut-line under OFF-state with (b) LIGUD = $2 \mathrm{~nm}$ and (c) LIGUD = $10 \mathrm{~nm}$; (d) Energy band along B-B' cut-line under ON-state at LIGUD $=2 \mathrm{~nm}$. 

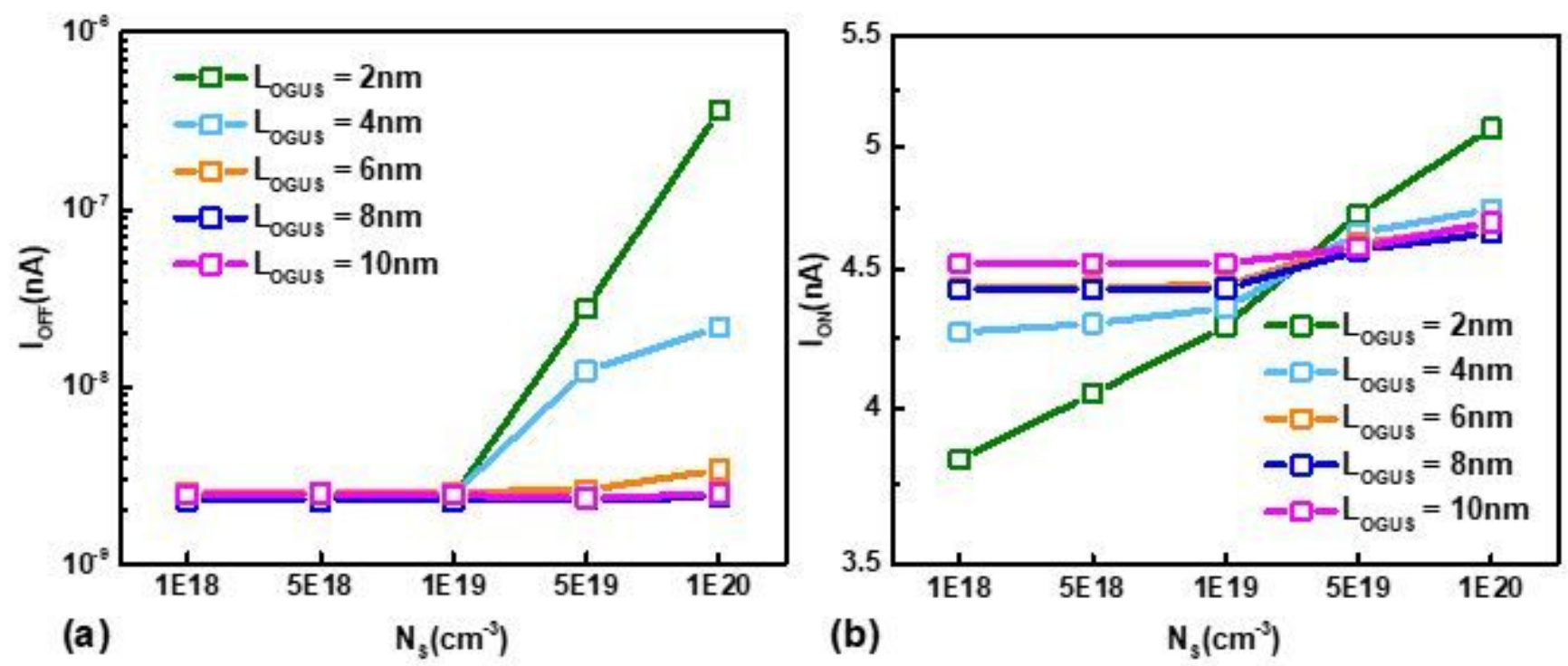

Figure 9

Impact of NS with various LOGUS on (a) IOFF and (b) ION for EHBNT-TFET.
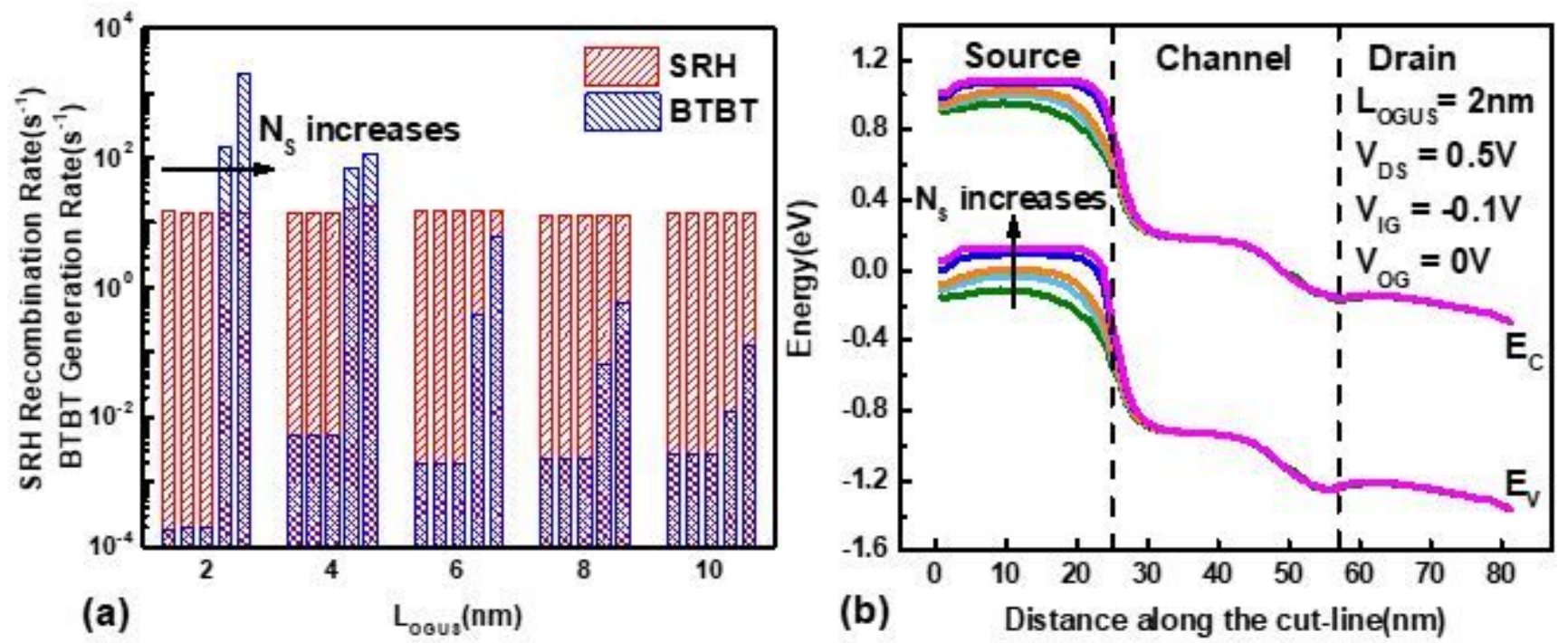

Figure 10

(a) SRH recombination rate and BTBT generation rate under OFF-state with various LOGUS for EHBNTTFET. (b) Energy band along B-B' cut-line under OFF-state for EHBNT-TFET. 

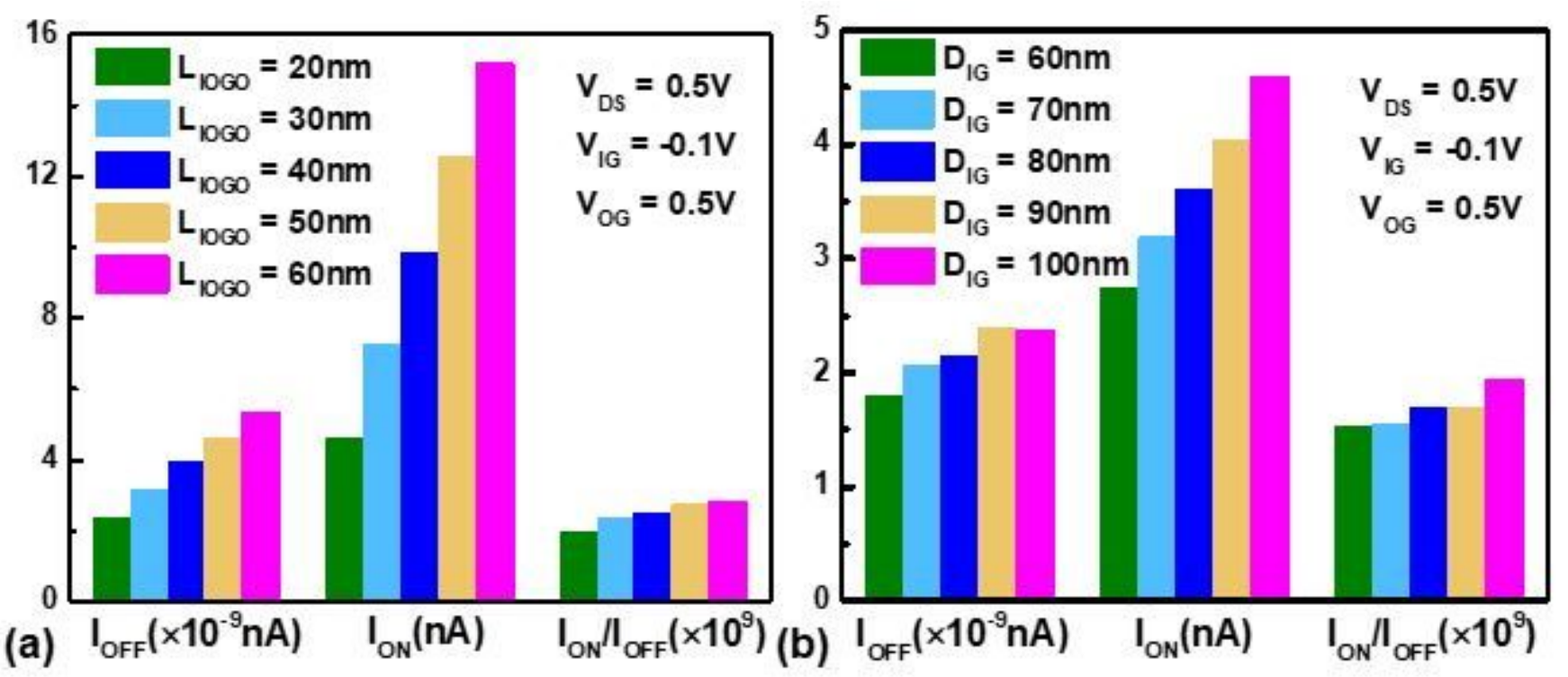

Figure 11

Impact of (a) LIOGO and (b) DIG on IOFF and ION for EHBNT-TFET.
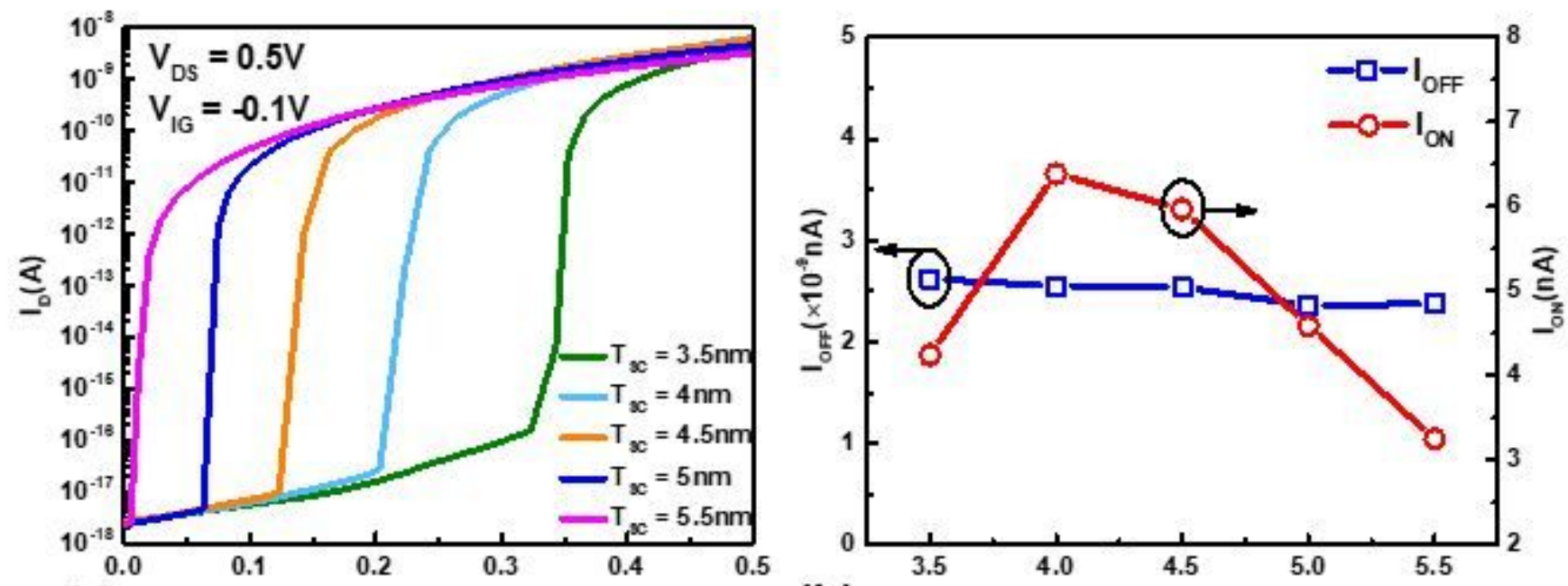

(a) $\quad \mathrm{v}_{\mathrm{og}}(\mathrm{V})$

(b)
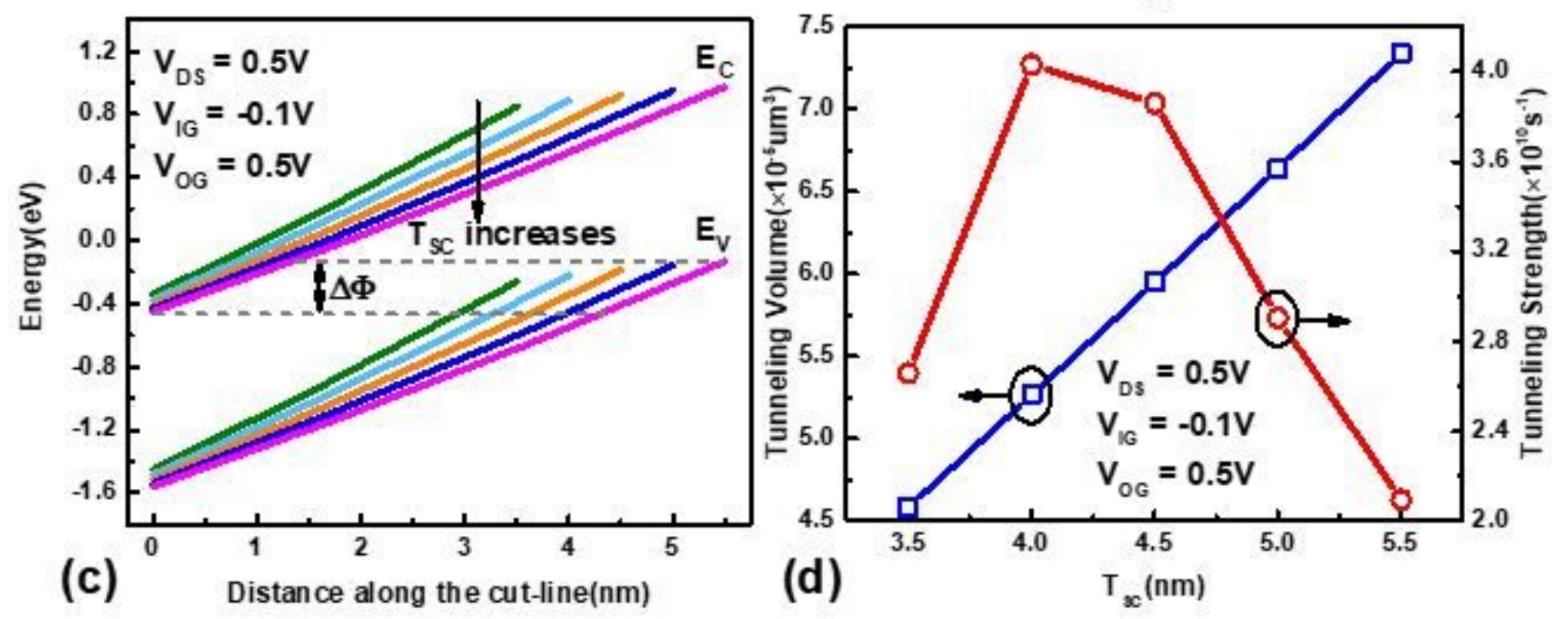

Loading [MathJax]/jax/output/CommonHTML/jax.js 
Figure 12

(a) Simulated transfer curve and (b) IOFF and ION with various TSC for EHBNT-TFET. (c) Energy band along $\mathrm{C}-\mathrm{C}^{\prime}$ cut-line and (d) tunneling volume and strength under ON-state for EHBNT-TFET.
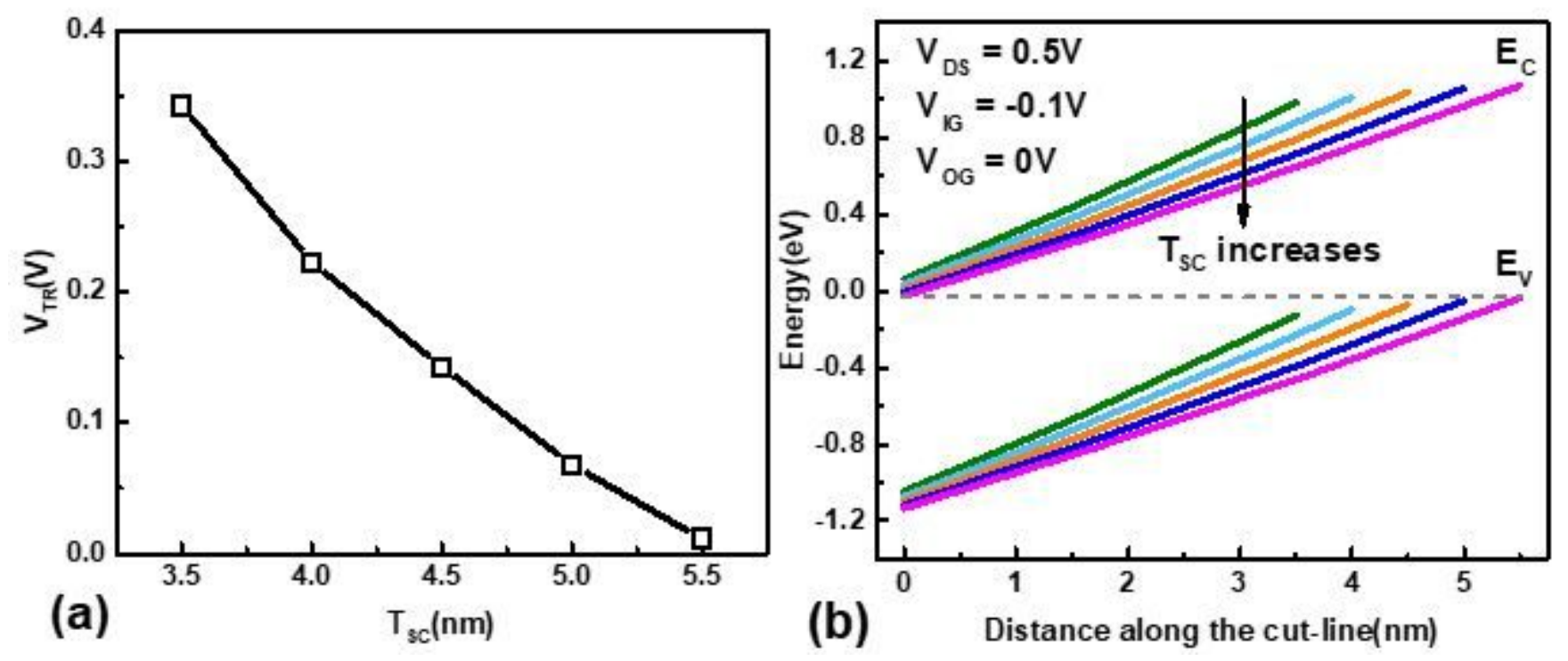

Figure 13

(a) VTR with various TSC for EHBNT-TFET. (b) Energy band along C-C' cut-line under OFF-state for EHBNTTFET. 

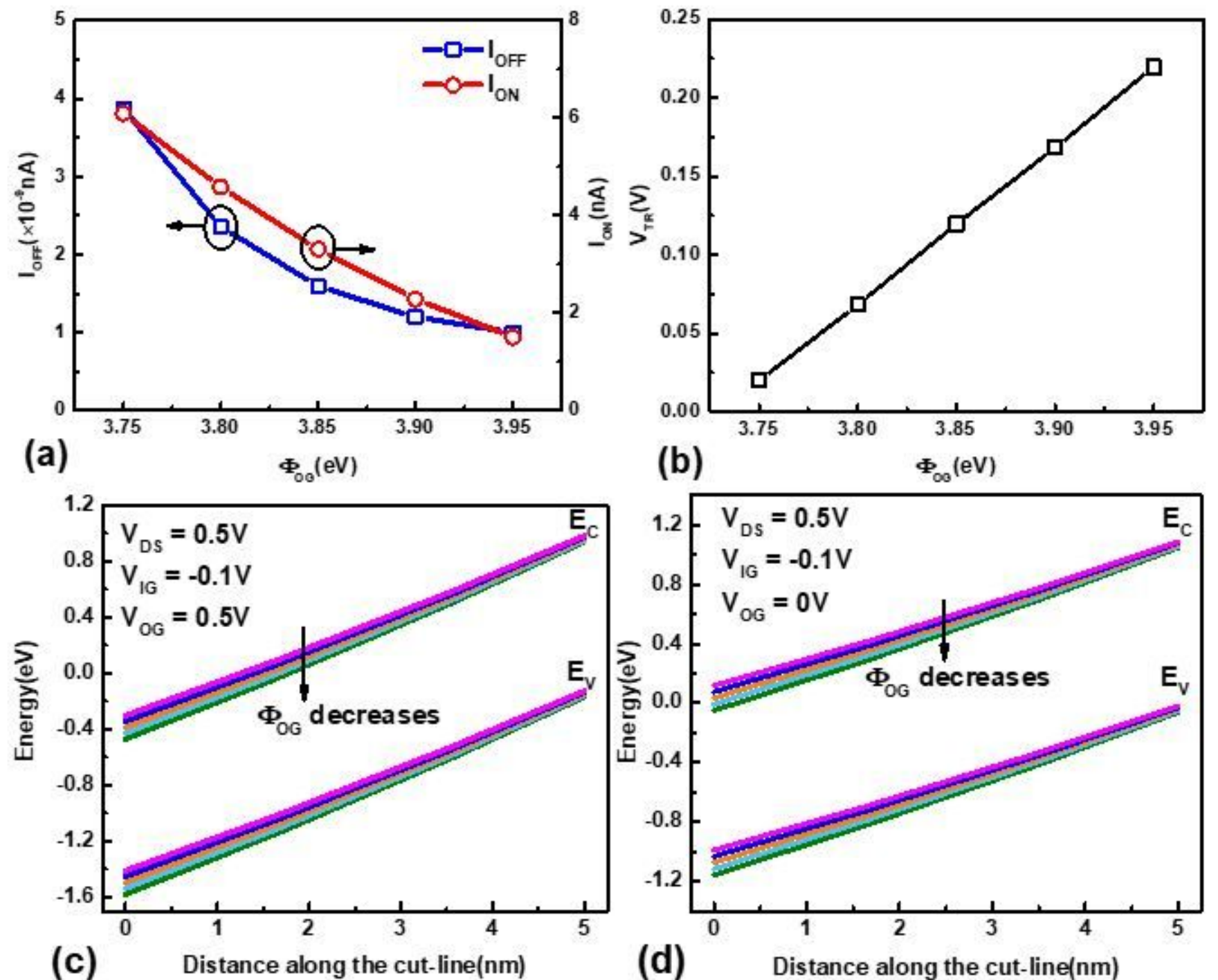

(b)

$\Phi_{\infty 0}(e V)$

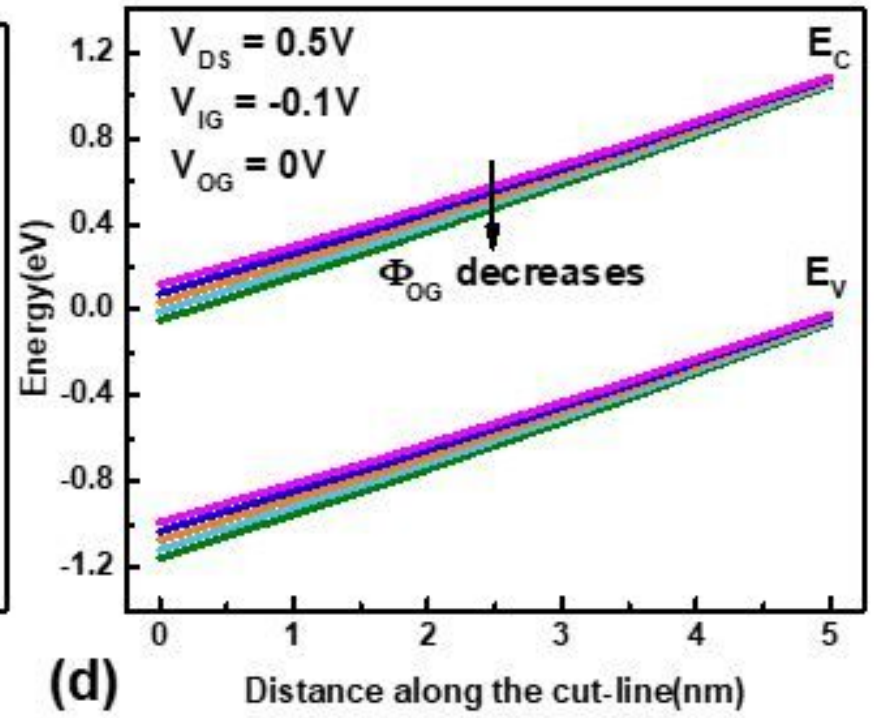

Figure 14

(a) IOFF and ION, (b) VTR, energy band along C-C' cut-line under (c) ON-state and (d) OFF-state for DOG variation of EHBNT-TFET. 

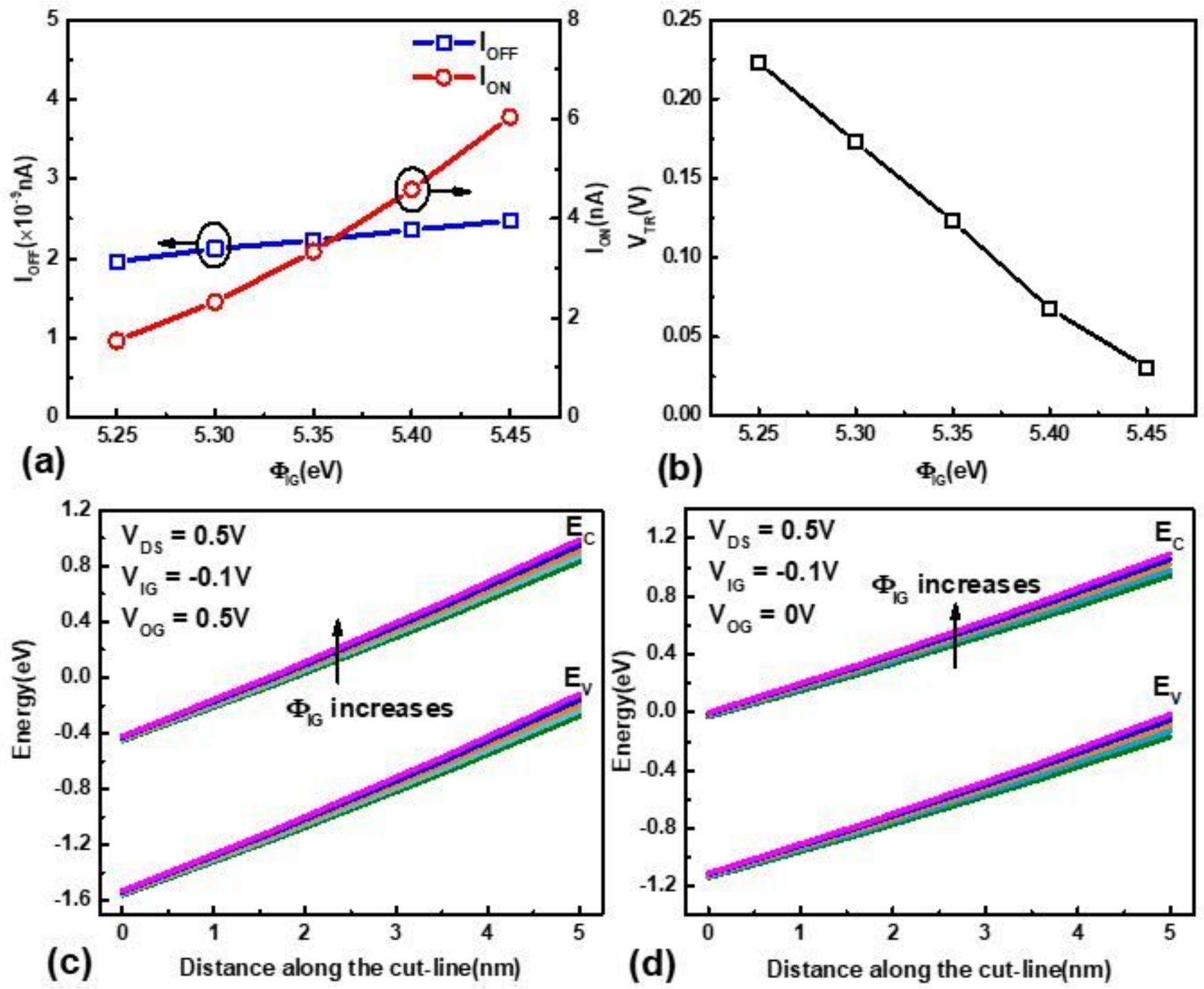

Figure 15

(a) IOFF and ION, (b) VTR, energy band along C-C' cut-line under (c) ON-state and (d) OFF-state for ФIG variation of EHBNT-TFET. 